\title{
Applicability of the DPPH Assay for Evaluating the Antioxidant Capacity of Food Additives - Inter-laboratory Evaluation Study -
}

\author{
Tomoko Shimamura, ${ }^{* 1 \dagger}$ Yoshihiro Sumikura, ${ }^{* 1}$ Takeshi Yamazaki, ${ }^{2, * 3}$ Atsuko Tada, ${ }^{* 2}$ \\ Takehiro KaShiwagi, ${ }^{* 1}$ Hiroya Ishikawa, ${ }^{* 4}$ Toshiro Matsui, ${ }^{* 5}$ Naoki Sugimoto, ${ }^{* 2}$ \\ Hiroshi AKIYAMA, ${ }^{* 2}$ and Hiroyuki UKEDA ${ }^{* 1}$ \\ *1 Faculty of Agriculture, Kochi University, Monobe B-200, Nankoku, Kochi 783-8502, Japan \\ *2 National Institute of Health Sciences, 1-18-1 Kamiyoga, Setagaya, Tokyo 158-8501, Japan \\ *3 Faculty of Human Life Sciences, Jissen Women's University, 4-1-1 Osakaue, Hino, Tokyo 191-8510, Japan \\ *4 International College of Arts and Sciences, Fukuoka Women's University, 1-1-1 Kasumigaoka, Higashi, \\ Fukuoka 813-8529, Japan \\ *5 Faculty of Agriculture, Graduated School of Kyushu University, 6-10-1 Hakozaki, Higashi, \\ Fukuoka 812-8581, Japan
}

\begin{abstract}
An inter-laboratory evaluation study was conducted in order to evaluate the antioxidant capacity of food additives by using a 1,1-diphenyl-2-picrylhydrazyl (DPPH) assay. Four antioxidants used as existing food additives (i.e., tea extract, grape seed extract, enju extract, and $d$ - $\alpha$-tocopherol) and 6-hydroxy-2,5,7,8-tetramethylchroman-2-carboxylic acid (Trolox) were used as analytical samples, and 14 laboratories participated in this study. The repeatability relative standard deviation $\left(\mathrm{RSD}_{\mathrm{r}}\right)$ of the $\mathrm{IC}_{50}$ of Trolox, four antioxidants, and the Trolox equivalent antioxidant capacity (TEAC) were $1.8-2.2 \%, 2.2-2.9 \%$, and $2.1-2.5 \%$, respectively. Thus, the proposed DPPH assay showed good performance within the same laboratory. The reproducibility relative standard deviation $\left(\mathrm{RSD}_{\mathrm{R}}\right)$ of $\mathrm{IC}_{50}$ of Trolox, four antioxidants, and TEAC were $4.0-7.9 \%, 6.0-11 \%$, and $3.7-9.3 \%$, respectively. The $\mathrm{RSD}_{\mathrm{R}} / \mathrm{RSD}_{\mathrm{r}}$ values of TEAC were lower than, or nearly equal to, those of $\mathrm{IC}_{50}$ of the four antioxidants, suggesting that the use of TEAC was effective for reducing the variance among the laboratories. These results showed that the proposed DPPH assay could be used as a standard method to evaluate the antioxidant capacity of food additives.
\end{abstract}

Keywords DPPH assay, inter-laboratory study, antioxidant, food additive

(Received April 22, 2014; Accepted May 23, 2014; Published July 10, 2014)

\section{Introduction}

In Japan, antioxidants are widely used as food additives in order to prevent oxidative rancidity and oxidative discoloration of fat and oil in food. Because the oxidative degradation of food constituents reduces the value of food, and ingestion of peroxides may have hazardous effects on the human body, antioxidants play an important role. Quality evaluation of antioxidants is crucial. ${ }^{1}$

The antioxidants commercially used in Japan are classified into designated additives or existing food additives. For designated additives, ingredient standards are established after their safety and availability have been validated. On the other hand, the use of existing food additives has been approved as a transitional measure since the revision of the Food Sanitation Act in 1995. Many existing food additives are complicated mixtures originating in nature, and the standards used as the index of known antioxidative compounds and/or compositions of these compounds need to be established. ${ }^{2}$ To establish the

† To whom correspondence should be addressed.

E-mail: tomokos@kochi-u.ac.jp standards for existing additives, verification of composition, identification of active compounds, and development of quantitative methods have been reported. ${ }^{3-4}$ However, it is often difficult to identify all the active compounds found in existing food additives. Thus, for antioxidants such as existing food additives, which do not have standards based on known antioxidant compounds, it seems to be necessary to propose a standard based on their antioxidant capacities to ensure quality. In a previous report, ${ }^{5}$ the candidates of standard methods for evaluating the antioxidant capacity of existing food additives were selected for the following reasons: (i) they have been widely applied in past studies, (ii) they can be performed within a short time, (iii) they are commonly-used spectrophotometric assays. Consequently, we selected the 1,1-diphenyl-2picrylhydrazyl (DPPH) assay and the 2,2'-azinobis(3ethylbenzothiazoline-6-sulfonic acid) (ABTS) assay among the methods used to measure the radical scavenging activity. In addition, the 2-(4-iodophenyl)-3-(4-nitrophenyl)-5-(2,4disulfophenyl)- $2 H$-tetrazolium (WST-1) assay was chosen among the methods used to measure the active oxygen species scavenging activity. In a small-scale collaborative study conducted at three laboratories, ${ }^{5}$ nine antioxidants (i.e., catechin, quercetin, sesamol, ferulic acid, gallic acid, morin, ellagic acid, 
$d$ - $\alpha$-tocopherol, and $d$ - $\delta$-tocopherol) were evaluated using the above-mentioned assays. The protocol of the DPPH assay was then improved based on the results of the small-scale collaborative study. Herein, the antioxidative activities of five analytical samples, four antioxidants used as existing food additives (i.e., tea extract, grape seed extract, enju extract and $d$ - $\alpha$-tocopherol), and 6-hydroxy-2,5,7,8-tetramethylchroman-2carboxylic acid (Trolox) were evaluated using the DPPH assay based on the improved protocol at fourteen laboratories. Thereafter, we investigated the validity of this assay as a standard method.

\section{Experimental}

\section{Participating laboratories}

This inter-laboratory evaluation study was conducted according to an international harmonized protocol. ${ }^{6}$ The fourteen laboratories that participated in this study was as follows: Food Analysis Technology Center SUNATEC; International College of Arts and Sciences, Fukuoka Women's University; Mycotoxin Research Association; Japan Food Research Laboratories; Faculty of Agriculture, Graduated School of Kyushu University; Japan Inspection Association of Food and Food Industry Environment; Japan Frozen Foods Inspection Corporation; College of Pharmacy, Kinjo Gakuin University; Faculty of Agriculture, Kochi University; Graduate School of Pharmaceutical Sciences, Nagoya City University; Division of Food Additives, National Institute of Health Sciences; College of Bioresources Science, Nihon University; Shin Nihon Kentei Kyokai; Tokyo Kenbikyoin Foundation. These laboratories were indicated as $\mathrm{A}$ to $\mathrm{N}$ in a random order. The analytical procedure listing the methods for preparing reagents and analytical samples, measuring, and the handling of data was distributed to the participating laboratories.

\section{Analytical samples}

Tea extract (main component: catechin), grape seed extract (main component: proanthocyanidin), enju extract (Japanese Pagoda Tree extract; main component: rutin), and $d$ - $\alpha$-tocopherol were obtained from the Japan Food Additive Association. One gram of the food additive sample from the same lot was distributed to each laboratory. The water solubility of active compounds in tea extract and grape seed extract is relatively high, and that in enju extract and $d$ - $\alpha$-tocopherol is relatively low. Based on the results of a preliminary experiment, tea extract and grape seed extract, which belonged to the class of high antioxidant capacity, enju extract, which belonged to the class of medium activity, and $d$ - $\alpha$-tocopherol, which belonged to the class of low activity, were selected as analytical samples among the various antioxidants of existing food additives.

Trolox of the same lot was purchased from Sigma-Aldrich (St. Louis, MO) and distributed to each laboratory without opening. The method used for storing analytical samples was detailed in the analytical procedure.

\section{DPPH radical scavenging assay}

In this study, the DPPH assay was conducted according to the following procedure. DPPH (Wako Pure Chemical Industries, Osaka, Japan) of the same lot was distributed to the participating laboratories. The methods for preparing each reagent were detailed in the analytical procedures. To prevent the influence of static electricity during the weighing of a reagent, a static electricity remover (EST-M, Ishiyama Seisakusyo Co., Ltd., Tokyo, Japan) was distributed to the participating laboratories.

\section{(1) Preparing DPPH solution}

DPPH (7.89 mg) was weighed on a chemical balance with a minimum weighing limit of $10 \mu \mathrm{g}$ or smaller. Thereafter, it was dissolved in $99.5 \%$ ethanol to obtain a constant volume by filling $100 \mathrm{~mL}$ of a measuring flask or a measuring cylinder with a stopper $(0.2 \mathrm{mM}$ DPPH). The absorbance of a DPPH solution is empirically known to decrease with time until approximately $1 \mathrm{~h}$ after preparation. Therefore, it was kept in the dark for $2 \mathrm{~h}$ until the absorbance stabilized. After $2 \mathrm{~h}, 1 \mathrm{~mL}$ of the DPPH solution was added into a test tube or sampling tube, and then $200 \mu \mathrm{L}$ of ethanol and $800 \mu \mathrm{L}$ of $0.1 \mathrm{M}$ Tris-HCl buffer ( $\mathrm{pH}$ 7.4) were added. After mixing, the absorbance at $517 \mathrm{~nm}$ was measured. A mixed solution containing $1.2 \mathrm{~mL}$ of ethanol and $800 \mu \mathrm{L}$ of Tris- $\mathrm{HCl}$ buffer was used as the blank. When the absorbance was in a range of $1.00 \pm 0.05$, the prepared DPPH solution was used directly for the measurements; when the absorbance exceeded 1.05 , ethanol was added to dilute the solution to be within a range of $1.00 \pm 0.05$. Afterwards, the solution was used for the measurements. The DPPH solution was stored in the dark at room temperature during the assay, and used up on the day of preparation.

\section{(2) DPPH assay procedure}

After $200 \mu \mathrm{L}$ of an analytical sample solution and $800 \mu \mathrm{L}$ of $0.1 \mathrm{M}$ Tris- $\mathrm{HCl}$ buffer ( $\mathrm{pH}$ 7.4) were added into a test tube or sampling tube, $1 \mathrm{~mL}$ of the DPPH solution was added. Immediately, the solution was mixed with a test tube mixer for $10 \mathrm{~s}$. Thereafter, it was left at room temperature in the dark. Exactly $30 \mathrm{~min}$ after the addition of the DPPH solution, the absorbance of the solution at $517 \mathrm{~nm}$ was measured. A mixed solution of $1.2 \mathrm{~mL}$ of ethanol and $800 \mu \mathrm{L}$ of Tris- $\mathrm{HCl}$ buffer was used as the blank.

The absorbance at the addition of the analytical sample was expressed as As, the absorbance at the addition of ethanol instead of the sample as Ac, and the inhibition ratio (\%) was obtained from the following equation:

$$
\text { Inhibition ratio }(\%)=\{(\mathrm{Ac}-\mathrm{As}) / \mathrm{Ac}\} \times 100 \text {. }
$$

In the analytical procedure distributed, the measurements at six points of concentration, including control, were required. The measurement of the DPPH radical scavenging activity for the analytical sample solution at each concentration was repeated three times.

\section{(3) Calculation of $I C_{50}$}

The $\mathrm{IC}_{50}$ of each analytical sample was calculated according to the following procedure:

(1) Inhibition ratios ( $y$ ) were plotted against the sample concentrations $(x)$ at all six points, and the respective regression line $(y=a x+b)$ was drawn.

The regression line was not required to pass through the origin. In this step, we verified that all of the measurement points were basically on the regression line. In addition, it was also verified that two points at around the 50\% inhibition did not have a deviation from the regression line. In fact, because the inhibition curve is not completely straight, but slightly curved, we decided to calculate the $\mathrm{IC}_{50}$ value using the interpolation method by joining the two points around the $50 \%$ inhibition with a straight line as follows:

(2) Two points enclosing a 50\% inhibition ratio were selected, and a regression line $(Y=\mathrm{A} X+\mathrm{B})$ was drawn. The regression line was not required to pass through the origin.

(3) $X$ (sample concentration) was calculated, when $Y$ in the regression equation of (2) was substituted with 50 . 
Table $1 \mathrm{IC}_{50}$ of antioxidants used as existing food additives determined using the DPPH assay in the inter-laboratory study

\begin{tabular}{|c|c|c|c|c|}
\hline \multirow[b]{2}{*}{ Laboratory } & \multicolumn{4}{|c|}{$\mathrm{IC}_{50} / \mu \mathrm{g} \mathrm{mL}^{-1 \mathrm{a}}$} \\
\hline & tea extract & $\begin{array}{c}\text { grape seed } \\
\text { extract }\end{array}$ & enju extract & $d$ - $\alpha$-tocopherol \\
\hline A & $27.0 \pm 0.4$ & $38.0 \pm 0.8$ & $76.6 \pm 0.4$ & $141 \pm 1.7$ \\
\hline B & $25.2 \pm 0.3$ & $33.7 \pm 1.8$ & $75.6 \pm 0.2$ & $139 \pm 5.4$ \\
\hline $\mathrm{C}$ & $25.7 \pm 1.0$ & $33.5 \pm 0.7$ & $73.9 \pm 1.5$ & $133 \pm 3.5$ \\
\hline $\mathrm{D}$ & $29.4 \pm 0.2$ & $32.7 \pm 0.2$ & $77.6 \pm 1.0$ & $137 \pm 0.8$ \\
\hline $\mathrm{E}$ & $24.2 \pm 0.4$ & $31.1 \pm 0.3$ & $77.8 \pm 0.9$ & $133 \pm 0.9$ \\
\hline $\mathrm{F}$ & $30.8 \pm 1.7$ & $36.1 \pm 0.8$ & $77.8 \pm 3.0$ & $142 \pm 3.0$ \\
\hline $\mathrm{G}$ & $25.3 \pm 0.8$ & $33.4 \pm 1.1$ & $74.1 \pm 0.5$ & $128 \pm 1.7$ \\
\hline $\mathrm{H}$ & $24.4 \pm 0.03$ & $33.5 \pm 0$ & $74.9 \pm 0.1$ & $134 \pm 0$ \\
\hline I & $26.2 \pm 0.6$ & $32.9 \pm 0.9$ & $78.2 \pm 0.5$ & $143 \pm 0.8$ \\
\hline $\mathrm{J}$ & $20.7 \pm 1.4$ & $31.4 \pm 0.4$ & $74.6 \pm 2.0$ & $148 \pm 6.4$ \\
\hline $\mathrm{K}$ & $25.3 \pm 0.1$ & $37.5 \pm 1.4$ & $76.4 \pm 4.5$ & $150 \pm 3.1$ \\
\hline $\mathrm{L}$ & $27.5 \pm 0.4$ & $35.9 \pm 0.6$ & $92.1 \pm 1.2$ & $137 \pm 1.6$ \\
\hline M & $25.7 \pm 0.8$ & $34.4 \pm 0.9$ & $75.3 \pm 3.7$ & $130 \pm 4.1$ \\
\hline $\mathrm{N}$ & $26.3 \pm 0.5$ & $31.5 \pm 0.5$ & $74.3 \pm 1.4$ & $135 \pm 1.7$ \\
\hline
\end{tabular}

a. Mean \pm S.D. $(n=3)$.

(4) The average value of (3) obtained at each of the three repetitive measurements was calculated. This value was set as the $\mathrm{IC}_{50}\left(\mu \mathrm{g} \mathrm{mL}^{-1}\right)$ value of each analytical sample.

\section{(4) Calculation of Trolox equivalent antioxidant capacity}

The DPPH radical scavenging activity of each analytical sample was expressed as the Trolox equivalent antioxidant capacity (TEAC). TEAC was calculated as follows:

TEAC $=\mathrm{IC}_{50}$ of Trolox $\left(\mu \mathrm{g} \mathrm{mL} \mathrm{L}^{-1}\right) / \mathrm{IC}_{50}$ of sample $\left(\mu \mathrm{g} \mathrm{mL}^{-1}\right)$.

The higher TEAC value means the higher DPPH radical scavenging activity. In this study, the values of the $\mathrm{IC}_{50}$ of an analytical sample and $\mathrm{IC}_{50}$ of Trolox, measured on the same day, were used to obtain the TEAC.

\section{Statistical Analyses}

Statistical analyses of the collected data were conducted as follows: ${ }^{6}$ outlier detection was conducted using the Cochran's test, single-Grubbs' test, and paired-Grubbs' test. The Cochran's test was used to identify the outlying variance. The single and paired-Grubbs' tests was used to identify the outlying value from the averages. Based on the result of each test, the outliers were rejected. An analysis of the variance was then conducted for each analytical sample so as to calculate the repeatability standard deviation $\left(S_{r}\right)$, reproducibility standard deviation $\left(S_{R}\right)$, repeatability relative standard deviation $\left(\mathrm{RSD}_{\mathrm{r}}\right)$, reproducibility relative standard deviation $\left(\mathrm{RSD}_{\mathrm{R}}\right)$, and $\mathrm{RSD}$ ratio $\left(\mathrm{RSD}_{\mathrm{R}} / \mathrm{RSD}_{\mathrm{r}}\right)$. Microsoft Office Excel 2010 was used for data analysis.

\section{Results}

Values reported by the participating laboratories

Tables 1 and 2 show the $\mathrm{IC}_{50}$ of the antioxidants $\left(\mu \mathrm{g} \mathrm{mL}^{-1}\right)$ and the $\mathrm{IC}_{50}$ of Trolox $\left(\mu \mathrm{g} \mathrm{mL}^{-1}\right)$, respectively, which were reported by the fourteen laboratories. In this study, one antioxidant and Trolox were measured on the same day, and the TEAC of each antioxidant was calculated based on the $\mathrm{IC}_{50}$ of Trolox obtained on the same day. Therefore, as shown in Table 2, Trolox measured on each day was dealt as an
Table $2 \mathrm{IC}_{50}$ of Trolox determined using the DPPH assay in the inter-laboratory study

\begin{tabular}{|c|c|c|c|c|}
\hline \multirow{3}{*}{ Laboratory } & \multicolumn{4}{|c|}{$\mathrm{IC}_{50} / \mu \mathrm{g} \mathrm{mL}^{-1}$ a } \\
\hline & \multicolumn{4}{|c|}{ Trolox } \\
\hline & tea extract & $\begin{array}{l}\text { grape seed } \\
\text { extract }\end{array}$ & enju extract & $d$ - $\alpha$-tocopherol \\
\hline A & $64.2 \pm 0.8$ & $64.3 \pm 0.7$ & $64.6 \pm 1.0$ & $61.3 \pm 1.2$ \\
\hline B & $57.9 \pm 2.0$ & $61.9 \pm 2.4$ & $61.4 \pm 2.2$ & $63.0 \pm 1.9$ \\
\hline $\mathrm{C}$ & $62.9 \pm 1.1$ & $60.8 \pm 1.4$ & $59.8 \pm 1.1$ & $58.6 \pm 1.6$ \\
\hline D & $65.9 \pm 0.08$ & $61.8 \pm 0.5$ & $63.5 \pm 0.5$ & $62.7 \pm 0.3$ \\
\hline E & $56.7 \pm 0.2$ & $57.6 \pm 0.2$ & $61.5 \pm 0.7$ & $61.8 \pm 0.8$ \\
\hline F & $61.2 \pm 1.1$ & $65.2 \pm 1.4$ & $62.7 \pm 0.5$ & $65.1 \pm 1.2$ \\
\hline G & $59.1 \pm 1.8$ & $59.1 \pm 1.9$ & $59.3 \pm 1.8$ & $58.5 \pm 0.7$ \\
\hline $\mathrm{H}$ & $56.4 \pm 0.1$ & $58.1 \pm 0.1$ & $58.5 \pm 0.1$ & $60.5 \pm 0.1$ \\
\hline I & $62.9 \pm 0.5$ & $61.2 \pm 1.0$ & $63.1 \pm 0.4$ & $61.9 \pm 0.7$ \\
\hline $\mathrm{J}$ & $55.9 \pm 1.2$ & $55.5 \pm 1.3$ & $61.2 \pm 0.4$ & $64.4 \pm 2.0$ \\
\hline $\mathrm{K}$ & $61.5 \pm 2.8$ & $50.6 \pm 1.3$ & $61.0 \pm 1.8$ & $63.0 \pm 1.7$ \\
\hline $\mathrm{L}$ & $64.1 \pm 0.8$ & $62.9 \pm 1.2$ & $70.1 \pm 1.0$ & $61.5 \pm 0.6$ \\
\hline M & $58.8 \pm 1.8$ & $61.4 \pm 1.8$ & $57.6 \pm 3.4$ & $58.3 \pm 1.6$ \\
\hline $\mathrm{N}$ & $59.1 \pm 1.3$ & $58.0 \pm 0.3$ & $59.3 \pm 0.3$ & $61.9 \pm 0.3$ \\
\hline
\end{tabular}

a. Mean \pm S.D. $(n=3)$

Table 3 TEAC of antioxidants used as existing food additives determined using the DPPH assay in the inter-laboratory study

\begin{tabular}{|c|c|c|c|c|}
\hline \multirow[b]{2}{*}{ Laboratory } & \multicolumn{4}{|c|}{ TEAC $^{a}$} \\
\hline & tea extract & $\begin{array}{c}\text { grape seed } \\
\text { extract }\end{array}$ & enju extract & $d$ - $\alpha$-tocopherol \\
\hline A & $2.38 \pm 0.04$ & $1.69 \pm 0.04$ & $0.84 \pm 0.004$ & $0.44 \pm 0.005$ \\
\hline B & $2.30 \pm 0.03$ & $1.84 \pm 0.10$ & $0.81 \pm 0.002$ & $0.45 \pm 0.02$ \\
\hline $\mathrm{C}$ & $2.45 \pm 0.10$ & $1.82 \pm 0.04$ & $0.81 \pm 0.02$ & $0.44 \pm 0.01$ \\
\hline D & $2.24 \pm 0.01$ & $1.89 \pm 0.009$ & $0.82 \pm 0.01$ & $0.46 \pm 0.003$ \\
\hline E & $2.34 \pm 0.04$ & $1.85 \pm 0.02$ & $0.79 \pm 0.009$ & $0.47 \pm 0.003$ \\
\hline $\mathrm{F}$ & $1.99 \pm 0.11$ & $1.81 \pm 0.04$ & $0.81 \pm 0.03$ & $0.46 \pm 0.01$ \\
\hline G & $2.34 \pm 0.07$ & $1.77 \pm 0.06$ & $0.80 \pm 0.006$ & $0.46 \pm 0.006$ \\
\hline $\mathrm{H}$ & $2.32 \pm 0.003$ & $1.73 \pm 0$ & $0.78 \pm 0.001$ & $0.45 \pm 0$ \\
\hline I & $2.40 \pm 0.06$ & $1.86 \pm 0.05$ & $0.81 \pm 0.005$ & $0.43 \pm 0.003$ \\
\hline $\mathrm{J}$ & $2.71 \pm 0.18$ & $1.77 \pm 0.03$ & $0.82 \pm 0.02$ & $0.44 \pm 0.02$ \\
\hline $\mathrm{K}$ & $2.44 \pm 0.008$ & $1.35 \pm 0.05$ & $0.80 \pm 0.05$ & $0.42 \pm 0.008$ \\
\hline $\mathrm{L}$ & $2.33 \pm 0.03$ & $1.76 \pm 0.03$ & $0.76 \pm 0.01$ & $0.45 \pm 0.005$ \\
\hline M & $2.29 \pm 0.07$ & $1.79 \pm 0.05$ & $0.77 \pm 0.04$ & $0.45 \pm 0.01$ \\
\hline $\mathrm{N}$ & $2.25 \pm 0.04$ & $1.85 \pm 0.03$ & $0.80 \pm 0.02$ & $0.46 \pm 0.006$ \\
\hline
\end{tabular}

a. Mean \pm S.D. $(n=3)$.

independent data point for statistical analysis. Table 3 gives the TEAC calculated from the values appearing in Tables 1 and 2 .

\section{Results of inter-laboratory study}

Tables 4 and 5 show the results of an inter-laboratory study on the $\mathrm{IC}_{50}$ of the antioxidants used as existing food additives and the $\mathrm{IC}_{50}$ of Trolox, respectively. Table 6 gives the results of the inter-laboratory study on TEAC.

\section{(1) $I C_{50}$ of the antioxidants used as existing food additives}

In the results on the $\mathrm{IC}_{50}$ of tea extract, grape seed extract, enju extract and $d$ - $\alpha$-tocopherol (Table 4 ), the result of enju extract measured by Laboratory $\mathrm{L}$ was determined as an outlier by a single-Grubbs' test. The reason was that the average of $\mathrm{IC}_{50}$ at Laboratory $\mathrm{L}$ was largest among all of the laboratories (Table 1), because the decrease ratio of the absorbance due to 
Table 4 Inter-laboratory study results for $\mathrm{IC}_{50}$ of antioxidants used as existing food additives

\begin{tabular}{|c|c|c|c|c|}
\hline & tea extract & grape seed extract & enju extract & $d$ - $\alpha$-tocopherol \\
\hline Number of laboratories & $14(0)$ & $14(0)$ & $13(1)^{\mathrm{a}}$ & $14(0)$ \\
\hline Grand mean $/ \mu \mathrm{g} \mathrm{mL}^{-1}$ & 26.0 & 34.0 & 75.9 & 138 \\
\hline Repeatability standard deviation $\left(\mathrm{S}_{\mathrm{r}}\right)$ & 0.76 & 0.88 & 2.02 & 3.05 \\
\hline Repeatability relative standard deviation (RSDr, \%) & 2.9 & 2.6 & 2.7 & 2.2 \\
\hline Reproducibility standard deviation $\left(\mathrm{S}_{\mathrm{R}}\right)$ & 2.96 & 2.76 & 2.38 & 8.27 \\
\hline Reproducibility relative standard deviation $\left(\mathrm{RSD}_{\mathrm{R}}, \%\right)$ & 11 & 8.1 & 3.1 & 6.0 \\
\hline $\mathrm{RSD}_{\mathrm{R}} / \mathrm{RSD}_{\mathrm{r}}$ & 3.9 & 3.1 & 1.2 & 2.7 \\
\hline
\end{tabular}

a. Laboratory $\mathrm{L}$ was determined as outlier.

Table 5 Inter-laboratory study results for $\mathrm{IC}_{50}$ of Trolox

\begin{tabular}{|c|c|c|c|c|}
\hline & \multicolumn{4}{|c|}{ Trolox } \\
\hline & tea extract & grape seed extract & enju extract & $d$ - $\alpha$-tocopherol \\
\hline Number of laboratories & $14(0)$ & $14(0)$ & $12(2)^{\mathrm{a}}$ & $14(0)$ \\
\hline Grand mean $/ \mu \mathrm{g} \mathrm{mL}^{-1}$ & 60.5 & 59.9 & 61.3 & 61.6 \\
\hline Repeatability standard deviation $\left(\mathrm{S}_{\mathrm{r}}\right)$ & 1.34 & 1.30 & 1.11 & 1.21 \\
\hline Repeatability relative standard deviation $\left(\mathrm{RSD}_{\mathrm{r}}, \%\right)$ & 2.2 & 2.2 & 1.8 & 2.0 \\
\hline Reproducibility standard deviation $\left(\mathrm{S}_{\mathrm{R}}\right)$ & 4.05 & 4.74 & 2.43 & 2.70 \\
\hline Reproducibility relative standard deviation $\left(\mathrm{RSD}_{\mathrm{R}}, \%\right)$ & 6.7 & 7.9 & 4.0 & 4.4 \\
\hline $\mathrm{RSD}_{\mathrm{R}} / \mathrm{RSD}_{\mathrm{r}}$ & 3.0 & 3.6 & 2.2 & 2.2 \\
\hline
\end{tabular}

a. Laboratory $\mathrm{L}$ and $\mathrm{M}$ were determined as outliers.

Table 6 Inter-laboratory study results for TEAC of antioxidants used as existing food additives

\begin{tabular}{lccc}
\hline & tea extract & grape seed extract & enju extract \\
\hline Number of laboratories & $12(2)^{\mathrm{a}}$ & $14(0)$ & $14(0)$ \\
Grand mean & 2.34 & 1.77 & 0.80 \\
Repeatability standard deviation $\left(\mathrm{S}_{\mathrm{r}}\right)$ & 0.05 & 0.04 & 0.02 \\
Repeatability relative standard deviation $\left(\mathrm{RSD}_{\mathrm{r}}, \%\right)$ & 2.1 & 2.5 & 2.5 \\
Reproducibility standard deviation $\left(\mathrm{S}_{\mathrm{R}}\right)$ & 0.09 & 0.17 & 0.03 \\
Reproducibility relative standard deviation $\left(\mathrm{RSD}_{\mathrm{R}}, \%\right)$ & 3.8 & 9.3 & 3.8 \\
RSD $_{\mathrm{R}} / \mathrm{RSD}_{\mathrm{r}}$ & 1.8 & 3.8 & 0.02 \\
\hline
\end{tabular}

a. Laboratory $\mathrm{F}$ and $\mathrm{J}$ were determined as outliers.

the addition of an analytical sample at Laboratory L was quite smaller than those at other laboratories on that day.

The $\mathrm{RSD}_{\mathrm{r}}$ of $\mathrm{IC}_{50}$ of the four antioxidants was $2.2-2.9 \%$, and $\mathrm{RSD}_{\mathrm{R}}$ was $3.1-11 \%$.

\section{(2) $I C_{50}$ of Trolox}

The results of Trolox evaluated on the same day as enju extract at Laboratories $\mathrm{L}$ and $\mathrm{M}$ were determined as outliers by a single-Grubbs' test and Cochran's test, respectively (Table 5). In the case of Laboratory $\mathrm{L}$, the reason for such a result was that its average $\mathrm{IC}_{50}$ was largest among all of the laboratories, because the decrease ratio of the absorbance due to the addition of an analytical sample at Laboratory $\mathrm{L}$ was quite smaller than those at other laboratories on that day. In the case of Laboratory $\mathrm{M}$, the reason was that the variance of the results of the three repetitive measurements was determined to be larger than those at other laboratories.

The $\mathrm{RSD}_{\mathrm{r}}$ of $\mathrm{IC}_{50}$ of Trolox was $1.8-2.2 \%$, and $\mathrm{RSD}_{\mathrm{R}}$ was $4.0-7.9 \%$.

\section{(3) TEAC}

The results of the TEAC of tea extract evaluated at Laboratory $\mathrm{F}$ and $\mathbf{J}$ were determined as outliers by single-Grubbs' test and Cochran's test, respectively (Table 6). In the case of Laboratory
F, the reason was that the average TEAC was smallest among all of the laboratories. This was caused by the fact that the $\mathrm{IC}_{50}$ of Trolox was an average value, whereas $\mathrm{IC}_{50}$ of tea extract measured on the same day was the largest among all of the laboratories. In the case of Laboratory J, the reason was that the variance of the results of three repetitive measurements was larger than those at other laboratories. This was caused by the fact that the variance of the $\mathrm{IC}_{50}$ of tea extract was relatively larger. On the other hand, the TEAC of enju extract measured at Laboratory $\mathrm{L}$, where both $\mathrm{IC}_{50}$ of enju extract and $\mathrm{IC}_{50}$ of Trolox on the same day were determined as outliers, did not result in an outlier. The reason seems to be that both $\mathrm{IC}_{50}$ of enju extract and Trolox were higher than those at other laboratories, which compensated in the conversion to the TEAC. The $\mathrm{RSD}_{\mathrm{r}}$ of TEAC was $2.1-2.5 \%$, and $\mathrm{RSD}_{\mathrm{R}}$ was $3.7-9.3 \%$.

\section{Discussion}

The $\mathrm{RSD}_{\mathrm{r}}$ of both $\mathrm{IC}_{50}$ and TEAC was smaller than $3.0 \%$, which showed that the proposed DPPH assay had a high repeatability within the same laboratory. In general, the RSD ratio of a chemical analysis is expected to be $1.5-2 .^{7}$ Although most of the RSD ratios were larger than 2 for the results of $\mathrm{IC}_{50}$ (Tables 4 
and 5), the RSD ratios of tea extract, enju extract, and $d$ - $\alpha$-tocopherol were smaller than 2 concerning the result of TEAC (Table 6). This result suggests that the use of the TEAC, a value calculated from the $\mathrm{IC}_{50}$ of the antioxidant and $\mathrm{IC}_{50}$ of Trolox measured on the same day, was effective for reducing the inter-laboratory differences by compensating for the variations.

Although many inter-laboratory evaluation studies on standard methods for food components have been conducted, there are few reports on a large-scale evaluation study on antioxidant capacity assays. ${ }^{8}$ In the case of the inter-laboratory collaborative study on improved Hydrophilic Oxygen Radical Absorbance Capacity (H-ORAC) assay, in which 14 laboratories participated, and (+)-catechin, ferulic acid, caffeine acid, hesperetin, Trolox, and five kinds of food (i.e., cabbage, onion, apple, Satsuma orange, and eggplant) were used as analytical samples, it was reported that the $\mathrm{RSD}_{\mathrm{r}}$ of the H-ORAC value $(\mu \mathrm{mol}$ Trolox/L) was $4.6-19 \%$, and the $\mathrm{RSD}_{\mathrm{R}}$ was $7.0-21 \% .{ }^{9}$ As the result of evaluating further improved $\mathrm{H}-\mathrm{ORAC}$ assay at five laboratories using similar samples, it was reported that the $\mathrm{RSD}_{\mathrm{r}}$ was $1.8-9.4 \%$, and the $\mathrm{RSD}_{\mathrm{R}}$ was $4.4-14 \% .^{9}$ An inter-laboratory collaborative study on the DPPH assay for 11 kinds of food and beverages at nine laboratories was also reported. ${ }^{10}$ In the DPPH assay used in the previous report, ${ }^{10}$ the DPPH concentration was $0.1 \mathrm{mM}$, the volume of the reaction solution was approximately $50 \mathrm{~mL}$, the reaction time was $4 \mathrm{~h}$, and the reaction temperature was $35^{\circ} \mathrm{C}$; those reaction conditions were largely different from those used in this study. As a result, it was reported that the $\mathrm{RSD}_{\mathrm{r}}$ of the antioxidant capacity ( $\mu \mathrm{mol}$ Trolox/100 g) was $1.1-25 \%$, and $\mathrm{RSD}_{\mathrm{R}}$ was $5.3-59 \% .^{10}$ Since the measurement principle of the DPPH assay was different from that of the ORAC assay, and the procedure and nature of the analytical samples in the DPPH assay used in the previous report were largely different from those in our study, the results of the two studies cannot be directly compared. However, the repeatability and reproducibility obtained in our study was found to be higher than those in the previous two reports. ${ }^{9,10}$

Herein, the absorbance was measured with spectrophotometers owned by the laboratories. Therefore, the equipment type was not unified. Although one of the laboratories used a shipper unit to measure the absorbance, neither the difference in types of spectrophotometers nor the difference in absorbance measuring methods had any influence on the results of this study. Therefore, the proposed DPPH assay had robustness not to be affected by types of the spectrophotometer.

As mentioned above, we selected antioxidants having different water solubility and antioxidant capacity based on the results of a preliminary experiment. The inter-laboratory evaluation revealed that the difference in the water solubility and antioxidant capacity of antioxidative compounds did not have a large influence on the precision of the proposed DPPH assay. Therefore, the proposed DPPH assay is applicable for evaluating the antioxidant capacity of a wide range of antioxidants.

Based on the above reasons, we concluded that the protocol of the DPPH assay used in this study is applicable as a standard method to evaluate the antioxidant capacity of antioxidants used as existing food additives. From now on, the antioxidative capacities of other existing food additives will be continuously collected to establish standard values for individual antioxidants based on their antioxidant capacities.

\section{Acknowledgements}

We expressed our deepest appreciation to all of the participating laboratories in this study. This study was supported by a Grant-in-Aid for Scientific Research from the Ministry of Health, Welfare and Labour of Japan.

\section{References}

1. H.-D. Belitz, W. Grosch, and P. Schieberle, "Food Chemistry", 4th ed., 2009, Springer-Verlag GmbH, Heidelberg, 187.

2. K. Sato, Shokuhin Eiseigaku Zasshi, 2010, 51, 331.

3. T. Yoshida, K. Terasaka, S. Kato, F. Bai, N. Sugimoto, H. Akiyama, T. Yamazaki, and H. Mizukami, Chem. Pharm. Bull., 2013, 61, 1264.

4. K. Ishizuki, A. Tada, N. Sugimoto, K. Matsumoto, H. Ukeda, H. Matsufuji, T. Yamazaki, and Y. Kawamura, Jpn. J. Food Chem. Safety, 2010, 17, 192.

5. T. Shimamura, R. Matsuura, T. Tokuda, N. Sugimoto, T. Yamazaki, H. Matsufuji, T. Matsui, K. Matsumoto, and H. Ukeda, Nippon Shokuhin Kagaku Kogaku Kaishi, 2007, 54, 482.

6. W. Horwitz, Pure Appl. Chem., 1995, 67, 331.

7. W. Horwitz, L. R. Kamps, and K. W. Boyer, J. AOAC Int., 1980, 63, 1344.

8. A. Hakoda, Nippon Shokuhin Kagaku Kogaku Kaishi, 2010, 57, 134 .

9. J. Watanabe, T. Oki, J. Takebayashi, K. Yamasaki, Y. Takano-Ishikawa, A. Hino, and A. Yasui, Anal. Sci., 2012, 28, 159.

10. D. W. Plank, J. Szpylka, H. Sapirstein, D. Woollard, C. M. Zapf, V. Lee, C. Y. Chen, R. H. Liu, R. Tsao, A. Düsterloh, and S. Baugh, J. AOAC Int., 2012, 95, 1562. 